\title{
On the Shape of the Galactic Dark Matter Halo
}

\author{
Amina Helmi $i^{1,2,3}$ \\ ${ }^{1}$ Astronomical Institute Utrecht, 3508 TA Utrecht, The Netherlands \\ 2 Kapteyn Institute, 9700 AV Groningen, The Netherlands \\ ${ }^{3}$ E-mail: ahelmi@astro.rug.nl
}

Received 2003 October 24, accepted 2004 March 22

\begin{abstract}
The confined nature of the debris from the Sagittarius dwarf to a narrow trail on the sky has recently prompted the suggestion that the dark matter halo of our Galaxy should be nearly spherical (Ibata et al. 2001; Majewski et al. 2003). This would seem to be in strong contrast with predictions from cold dark matter (CDM) simulations, where dark halos are found to have typical density axis ratios of 0.6 to 0.8 . Here I present numerical simulations of the evolution of a system like the Sagittarius dSph in a set of Galactic potentials with varying degrees of flattening. These simulations show that the Sagittarius streams discovered so far are too young dynamically to be sensitive to the shape of the dark halo of the Milky Way. The data presently available are entirely consistent with a Galactic dark matter halo that could either be oblate or prolate, with density axis ratios $c / a$ that range from 0.6 to 1.6 within the region of the halo probed by the orbit of the Sagittarius dwarf.
\end{abstract}

Keywords: dark matter — galaxies: halos, shapes — Galaxy: halo, dynamics, structure

\section{Introduction}

The shapes of dark matter halos are sensitive to the nature of dark matter itself. Flattened halos, more often prolate than oblate, are generally found in cold dark matter simulations (e.g. Dubinski \& Carlberg 1991); more spherical halos are characteristic of self-interacting or hot dark matter (Yoshida et al. 2000; Davé et al. 2001; Mayer et al. 2002). If dark matter was baryonic, then the halos are expected to be strongly flattened, with a ratio of minor to major axis of about 0.2 (Pfenninger \& Combes 1994). If, on the other hand, the observed flat rotation curves are due to a modified law of gravity, such as MOND, then the 'fictitious dark component' ought to be equivalent to a spherical (massless) halo (Milgrom 2001).

Some of the best probes of the shape of dark halos are tidal streams, since they consist of stars moving on very similar orbits (Lynden-Bell \& Lynden-Bell 1995; Johnston 1998). Essentially, the rate of divergence of their orbits depends not only on the internal properties of the progenitor satellite but also, and more specifically, on the shape of the host potential.

As an application of this idea, Ibata et al. (2001) have suggested that the recently discovered streams from the Sagittarius dwarf, favour a spherical dark halo, $q_{\rho} \sim 1$, for the Milky Way. Halo-flattening values of $q_{\rho} \leq 0.7$ are rejected at very high confidence levels (less than one chance in a million). This result is particularly important, because it would seem to be at odds with the measurements of the shapes of dark halos in $\mathrm{N}$-body CDM simulations, where typically the density (or mass) axis ratios $q_{\rho}$ are of the order of $0.6-0.8$ (Frenk et al. 1988; Thomas et al. 1998; Bullock 2002).
I present here numerical simulations in an attempt to model the evolution of the Sgr dwarf galaxy and of its debris as it orbits the Milky Way. The goal is to determine whether the presently available data on the debris from Sgr can constrain the shape of the Galactic halo as strongly as has been suggested in previous work.

\section{The Simulations}

In the numerical simulations, the Galaxy is represented by a fixed potential with three components: a dark logarithmic halo

$$
\Phi_{\text {halo }}=v_{\text {halo }}^{2} \ln \left(R^{2}+z^{2} / q^{2}+d^{2}\right),
$$

a Miyamoto-Nagai disk

$$
\Phi_{\text {disk }}=-\frac{G M_{\mathrm{disk}}}{\sqrt{R^{2}+\left(a+\sqrt{z^{2}+b^{2}}\right)^{2}}},
$$

and a spherical Hernquist bulge

$$
\Phi_{\text {bulge }}=-\frac{G M_{\text {bulge }}}{r+c},
$$

where $d=12 \mathrm{kpc}$ and $v_{\text {halo }}=131.5 \mathrm{~km} \mathrm{~s}^{-1} ; \quad M_{\text {disk }}=$ $10^{11} \mathrm{M}_{\odot}, a=6.5 \mathrm{kpc}$ and $b=0.26 \mathrm{kpc} ; M_{\text {bulge }}=3.4 \times$ $10^{10} \mathrm{M}_{\odot}$ and $c=0.7 \mathrm{kpc}$. The parameter $q$ is allowed to vary from 0.8 to 1.25 , that is, from an oblate to a prolate configuration. Figure 1 shows the variation of the density flattening for the halo component in these models (black curves), as well as for the global potential (light grey curves). Here $q_{\rho}$ is defined by the ratio of the distances down the $z$ and $R$ axes at which a given isodensity surface cuts those axes (see Binney \& Tremaine 1987, p. 48). 
The orbit of Sagittarius is relatively well constrained (Ibata et al. 1997). To set up the simulations I generated random orbits consistent with these constraints. The Sgr galaxy was represented by a collection of $5 \times 10^{4}$ particles, and their self-gravity modelled by a multipole expansion of the internal potential to the fourth order (White 1983; Zaritsky \& White 1988). These particles

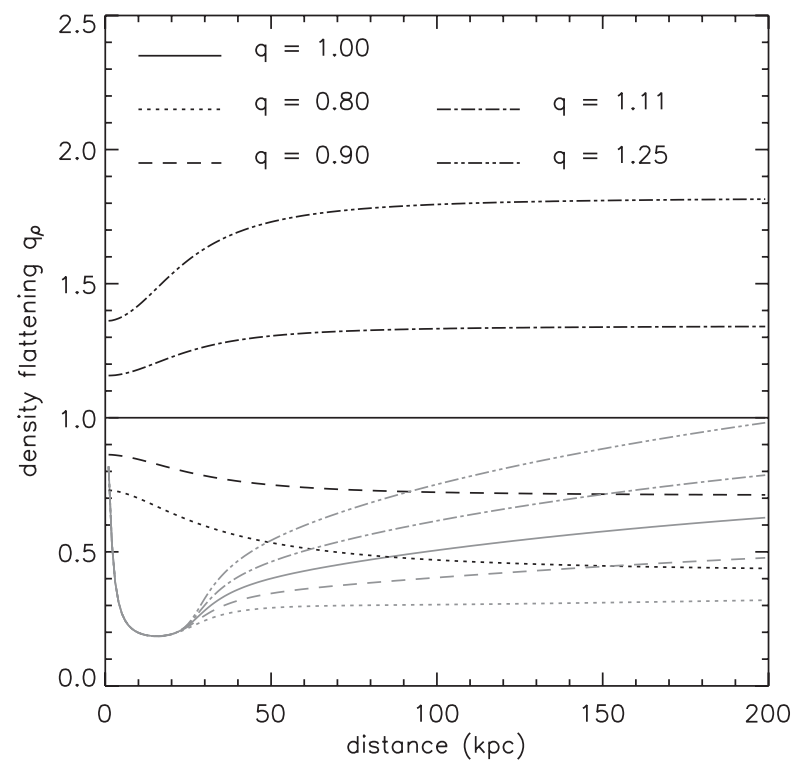

Figure 1 The variation of the density flattening, measured by the density axis ratio, as a function of distance from the centre of the galaxy for our model of the Milky Way, for the halo component (black) and for the global potential (grey). were initially distributed according to a King model, similar to the stellar model of Helmi \& White (2001). To make a proper comparison of the effect that the variation in the degree of flattening of the halo component has on the evolution of the debris, I imposed two strong conditions. On the one hand, I required that the orbits of the centre of mass in all cases have similar mean apocentric and pericentric distances as well as $L_{z}$. On the other hand, the initial properties of the dwarf were tuned so that, in all cases, the system would reach a similar degree of disruption, typically $10-20 \%$ of the initial mass remains bound after $10 \mathrm{Gyr}$ of evolution.

\section{Results}

The panels in Figure 2 show the sky distribution in equatorial coordinates (RA, DEC) for all the particles in the different experiments. The particles are colour-coded according to when they became unbound from their parent satellite. Dark grey colouring corresponds to particles unbound in the first $3.5 \mathrm{Gyr}$ of evolution (approximately five pericentric passages), light grey to those released between 3.5 and 7 Gyr (between approximately five and nine passages), and black to those that have been released in the last $3 \mathrm{Gyr}$ of evolution (or less than four passages ago). This last subset also includes particles that are still bound, i.e. what we call the main body of Sgr.

It is immediately striking that the distribution of black particles is very similar in all five cases. The particles define relatively narrow structures on the sky. These streams are so young dynamically that they have not really
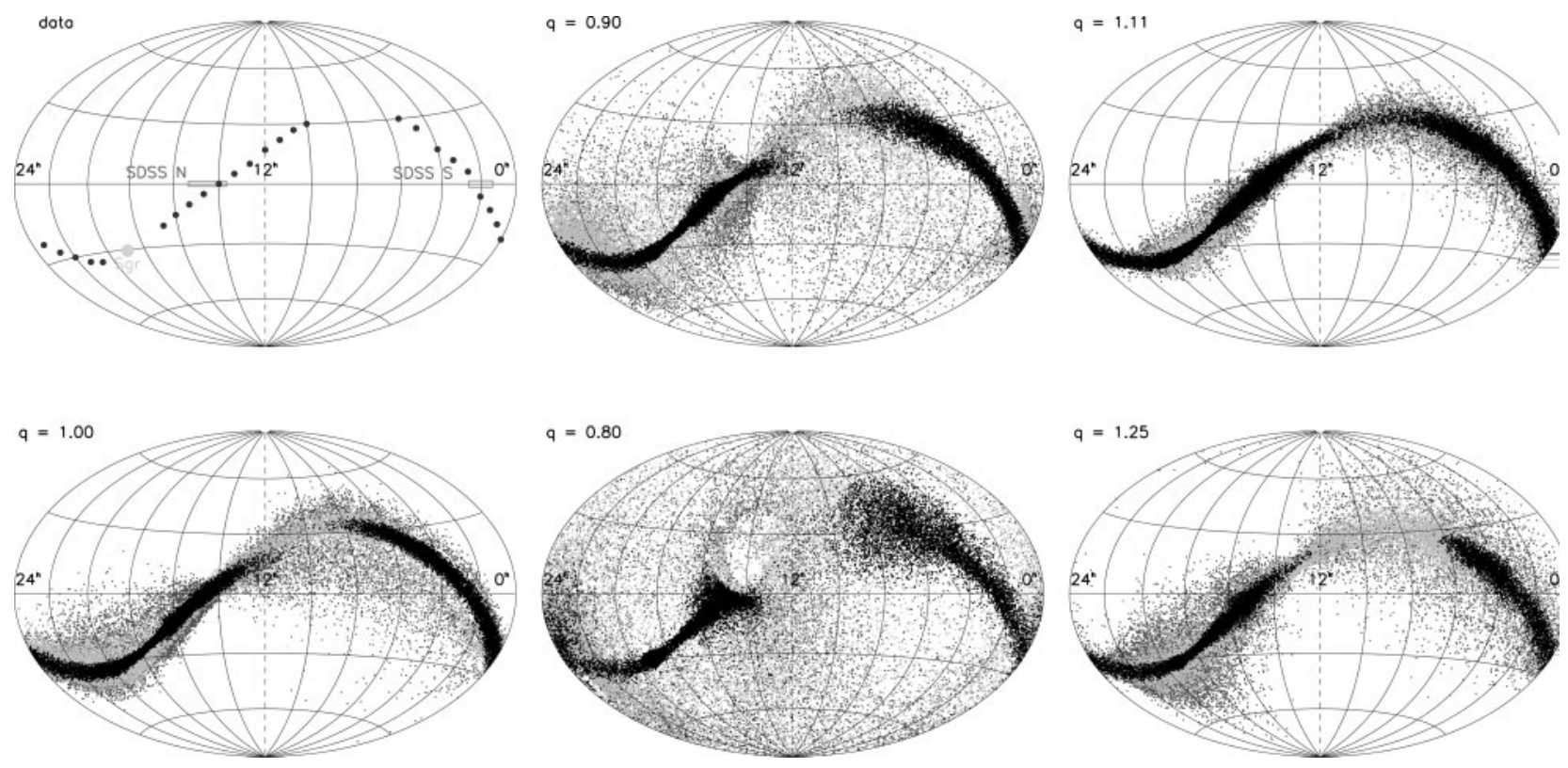

Figure 2 The different panels show the sky distribution (RA, DEC) of particles in our numerical simulations with varying degrees of flattening for the dark halo of the Galaxy. The different colours represent sets of particles which have become unbound more than $6.5 \mathrm{Gyr}$ ago (dark grey), more than $3 \mathrm{Gyr}$ but less than $6.5 \mathrm{Gyr}$ ago (light grey), and less than $3 \mathrm{Gyr}$ ago (black). In the top left panel, the different detections of debris from Sgr are shown. Here the black solid dots represent approximately the 2MASS M-giants stream (Majewski et al. 2003), while the boxes correspond to the northern and southern detections by SDSS (Ivezic et al. 2000; Yanny et al. 2000), which overlap substantially with detections by other groups. Direct comparison of the first panel with any of our numerical experiments suggests that the observations could be tracing only the most recently formed streams of Sgr (black dots in the different panels). 
been able to experience the flattening of the dark halo in which they orbit.

For comparison, the first panel of Figure 2 shows the distribution of claimed detections of debris from Sgr discovered so far by the SDSS (Ivezic et al. 2000; Yanny et al. 2000) and by Majewski et al. (2003). There seems to be a very good correspondence between the location of this debris and that of the most recently lost particles in our simulations (black dots in the remaining panels). These results would seem to imply that the observed streams could be, in fact, made up of stars lost by the Sgr dwarf in the last $3 \mathrm{Gyr}$ of evolution. If this were the case, the immediate conclusion would be that such observations cannot put meaningful constraints on the shape of the Galactic halo.

But are the black dots in Figure 2 really tracing all the debris that has been discovered thus far? To determine whether this is the case, we also needed to check that their dynamical properties (radial velocities and spatial location) are indeed similar. To that end, Figure 3 shows the distance and radial velocity (top and bottom panels, respectively) versus right ascension distribution of particles for one of the experiments, that with a prolate halo of $q=1.11$. We have used the same colour-coding as before, and also overplotted the detections of the northern and southern Sgr debris discovered by SDSS. It becomes now clear that these detections are all tracing only the material that has been released by the Sgr dwarf in the last 3 Gyr.

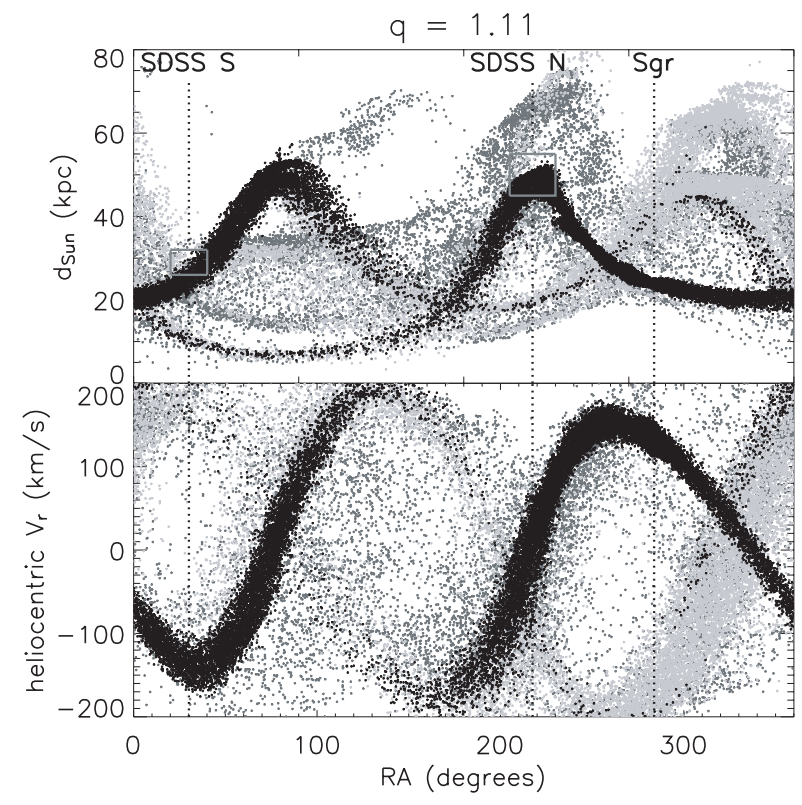

Figure 3 The top panel shows the distance versus right ascension distribution of particles in our numerical simulation with $q=1.11$. The same colour-coding as in Figure 2 is used. The bottom panel shows the radial velocity versus right ascension distribution for the same simulation. The northern and southern detections of debris from Sgr by SDSS are shown by the light grey boxes. Note that these share the same spatial distribution (in the sky and in distance) as well as kinematics of the most recently unbound material, indicated here by the black solid dots. This confirms that the streams discovered thus far are dynamically young.
All experiments of different flattening essentially show the same features, and are in agreement with the observational data available about Sgr and its streams.

We also note here that the tidal streams traced by the 2MASS M-giants in Majewski et al. (2003), are also well represented by the black dots shown in Figures 2 and 3 .

\section{Discussion}

Our numerical experiments of the evolution of the Sgr dwarf and its debris orbiting in Galactic potentials with different degrees of flattening show that dynamically young streams (younger than about $3 \mathrm{Gyr}$ ) on a Sgr-like orbit remain very coherent spatially, defining great circle arcs on the sky even when the density axis ratio of the dark halo of our Galaxy model is as low as 0.6 or as high as 1.6 (where this is the mean halo flattening in the region probed by the orbit of the Sgr dwarf). A straightforward comparison to the various detections of debris material from Sgr leads to the conclusion that this debris is dynamically young. Hence its striking confinement to a great circle arc on the sky does not imply that the dark halo of our Galaxy is nearly spherical.

A direct comparison to Ibata et al. (2001) models is possible, particularly for their $q_{m}=0.75$ case. This massdensity flattening is similar to our $q=0.9$ example (where we find $\left\langle q_{\rho}\right\rangle=0.78$ within the orbit). It is reassuring that the distribution of particles is very similar in both his as our simulation, if no distinction is made between the times the different particles were released. It then becomes clear that considering the dynamically young streams is crucial for a fair comparison to the observations.

To measure the shape of the dark halo of the Galaxy reliably dynamically old streams are required, made up of material lost more than 5 Gyr ago in the case of the Sgr dwarf. This, of course, implies using stars that trace old stellar populations. Such older streams will typically have very low surface brightness, and hence are more difficult to detect, particularly if the dark halo of our Galaxy is not spherical.

\section{Acknowledgments}

I would like to thank Brad Gibson for a very enjoyable conference and for the hospitality during my visit to Swinburne.

\section{References}

Binney, J., \& Tremaine, S. 1987, Galactic Dynamics (Princeton: Princeton University Press)

Bullock, J. S. 2002, in Proceedings of the Yale Cosmology Workshop 'The Shapes of Galaxies and Their Dark Matter Halos', ed. P. Natarajan (Singapore: World Scientific), p. 109

Davé, R., Spergel, D. N., Steinhardt, P. J., \& Wandelt, B. D. 2001, ApJ, 547, 574

Dubinski, J., \& Carlberg, R. G. 1991, ApJ, 378, 496

Frenk, C. S., White, S. D. M., Davis, M., \& Efstathiou, G. 1988, ApJ, 327, 507

Helmi, A. 2003, submitted to MNRAS (astro-ph/0309579)

Helmi, A., \& White, S. D. M. 2001, MNRAS, 323, 529 
Ibata, R. A., Wyse, R. F. G., Gilmore, G., Irwin, M. J., \& Suntzeff, N. B. 1997, AJ, 113, 634

Ibata, R., Lewis, G., Irwin, M., Totten, E., \& Quinn, T. 2001, ApJ, 551,294

Ivezic, Z., et al. (SDSS), 2000, AJ, 120, 963

Johnston, K. V. 1998, ApJ, 495, 297

Lynden-Bell, D., \& Lynden-Bell, R. M. 1995, MNRAS, 275, 429

Majewski, S., Skrutskie, M. F., Weinberg, M. D., \& Ostheimer, J. C. 2003, ApJ, 599, 1082

Mayer, L., Moore, B., Quinn, T., Governato, F., \& Stadel, J. 2002, MNRAS, 336, 119
Milgrom, M. 2001, MNRAS, 326, 1261

Pfenniger, D., \& Combes, F. 1994, A\&A, 285, 94

Thomas, P. A., et al. 1998, MNRAS, 296, 1061

White, S. D. M. 1983, ApJ, 274, 53

Yanny, B., et al. (SDSS), 2000, ApJ, 540, 825

Yoshida, N., Springel, V., White, S. D. M., \& Tormen, G. 2000, ApJ, 535, L103

Zaritsky, D., \& White, S. D. M. 1988, MNRAS, 235, 289 